\title{
DAMPNESS AND MOULD IN SCHOOL BUILDINGS AND RESPIRATORY SYMPTOMS IN TEACHERS: AN
} INTERNATIONAL STUDY

Jan-Paul Zock, ${ }^{1}$ Alicia Borràs-Santos, ${ }^{1}$ José Jacobs, ${ }^{2}$ Esmeralda Krop, ${ }^{2}$ Anne Hyvärinen, ${ }^{3}$ Martin Täubel, ${ }^{3}$ Ulla Haverinen-Shaughnessy, ${ }^{3}$ Dick Heederik, ${ }^{2}$ Juha Pekkanen, ${ }^{3}$ Aino Nevalainen ${ }^{3}$ CREAL, Barcelona, Spain, ${ }^{2}$ Utrecht University, Utrecht, The Netherlands; ${ }^{3} T H L$, Kuopio, Finland

10.1136/oemed-2011-100382.301

Objectives Moisture damage in homes has been associated with asthma and rhinitis, but the effect of such exposures in the workplace has not evaluated been in detail. We studied 
respiratory symptoms in teachers in relation to dampness and mould in schools.

Methods Primary schools in Finland, The Netherlands and Spain were selected according to the presence $(n=29)$ or absence $(n=26)$ of reported moisture damage (dampness and/or mould) that was confirmed by standardised building inspections. Teachers ( $\mathrm{n}=645,79 \%$ women) completed a questionnaire including work history and respiratory symptoms (ECRHS). The validated asthma symptom score was calculated as the number of reported asthma symptoms $(0-5)$ in the last year. Associations between moisture damage and symptoms were evaluated by using logistic and negative binomial regression analyses adjusting for sex, age, smoking status and country.

Results Teachers from exposed schools reported more asthma symptoms; the adjusted ratio of the mean asthma symptom score was 1.34 (95\% CI 1.01 to 1.78). This effect was stronger among teachers who had been working more than 5 years in the same school (1.60; 1.04 to 2.45) than among those employed 5 years or less (1.20; 0.81 to 1.76$)$. Work-related wheeze or chest tightness $(2.98 ; 1.78$ to 4.97$)$, and nasal symptoms $(1.58 ; 1.13$ to 2.21) were also more commonly reported by teachers from damaged schools. All findings were consistent across the three countries.

Conclusions Teachers from moisture damaged schools appear to be at increased risk of upper and lower respiratory tract symptoms, implying public health relevance of damp and mould problems in indoor workplaces. 